\title{
EEG Analysis during Light Stimulus based on Morphological Multiresolution Analysis
}

\author{
Tomonari Yamaguchi, Jun Irie, Mitsuhiko Fujio and Katsuhiro Inoue \\ Department of Systems Design and Informatics, Kyushu Institute of Technology \\ 680-4, Kawazu, Iizuka, Fukuoka 820-8502, Japan \\ E-mail: t_yamagu@kiri.ces.kyutech.ac.jp
}

\begin{abstract}
It is known that Electroencephalograph (EEG) signal shows specific responses according to the event (e.g. visual stimulus, cognition and motor imagery). Especially, by classifying short time EEG signal, features are used to control an electronic device (such system is called brain computer interface: BCI). Generally, in feature extraction from EEG signal, these features are extracted by using linear method such as FIR filter and wavelet transform etc. Though, linear method is not suitable because impulse noise distorts the important features. To avoid this, the morphological analysis method with non-linear characteristics has been used in this fields. In this paper, we propose a design method of structuring function that determine the filter characteristic of morphology. The morphological method is compared to discrete wavelet transform (DWT) from the view point of filter characteristics. We apply our method to real data observed from visual stimulation.
\end{abstract}

\section{Introduction}

It is known that Electroencephalograph (EEG) signal shows specific responses in the event (e.g. visual stimulus, cognition and motor imagery). This characteristic can be confirmed from averaged signal. Especially, by classifying short time EEG signal, features are used to control an electronic device (such system is called brain computer interface: $\mathrm{BCI}$ ) [5]. To construct BCI system, many methods have been proposed. For example, cursor control system had been proposed by using the feature that appeared in voluntary motor imagery such as hand movement [4][6]. Recently, many researchers studied about the P300 typewriter system which used cognitive response [1]. Though this system does not need training with user, this system requires a lot of time to distinguish (about 2-10 sec).

In this study, we tried to analyze feature of visual evoked potential since this phenomenon appears short latency. Generally, in feature extraction from EEG signal, these features are extracted by using linear method such as FIR filter and wavelet transform

"This work has been partially supported by the Grantin-Aid for Scientific Research (B) of Japan Society for the Promotion of Science, No. 21300208. etc. Though, linear method is not suitable because impulse noise distort the important features. To avoid this, the morphological analysis method with non-linear characteristics has been used in this fields [2]. Furthermore, since many elements compose this feature, it is necessary to decompose this signal by multi-resolution analysis (MRA). Therefore, we will identify significant component by using morphological MRA. This morphological decomposition is composed such as redundancy discrete wavelet transform (RDWT) [3] to avoid shift noise. The structuring function which decide the filter characteristic is designed to obtain optimal separation based on mutual information method or spectrum dividing method. The filter characteristics using these method and Haar type analysis method are compared to DWT based on Daubechies. We apply our method to real data observed from visual stimulation.

\section{Morphological MRA}

\subsection{Morphology}

In this section, we give a brief description on mathematical morphology. As basic operations, we employ Minkowski addition $\oplus$ and Minkowski subtraction $\ominus$, which are defined as follows.

$$
\begin{aligned}
& {[f \oplus g](t)=\max _{\substack{t-u \in F \\
u \in G}}\{f(t-u)+g(u)\}} \\
& {[f \ominus g](t)=\min _{u \in G}\{f(t-u)-g(u)\}}
\end{aligned}
$$

where $f(t)$ is an original signal and $g(t)$ is the structuring function which characterizes the filter. Furthermore, $F$ and $G$ denote the domains of $f(t)$ and $g(t)$, respectively. Conventionally, it is assumed that every signal takes the value $-\infty$ out of its domain. By combining these operations, we define two morphological filters:

$$
\begin{aligned}
\text { opening : } f_{g}(t) & =\left[\left(f \ominus g^{s}\right) \oplus g\right](t) \\
\text { closing : } f^{g}(t) & =\left[\left(f \oplus g^{s}\right) \ominus g\right](t)
\end{aligned}
$$

where $g^{s}(t):=g(-t)$. The opening process for $f(t)$ by $g(t)$ removes the parts in the positive direction of the wave form of $f(t)$ those are too narrow to fit for the wave form of $g(t)$ attached below. In contrast, the closing filter removes the narrow negatively directed parts. 


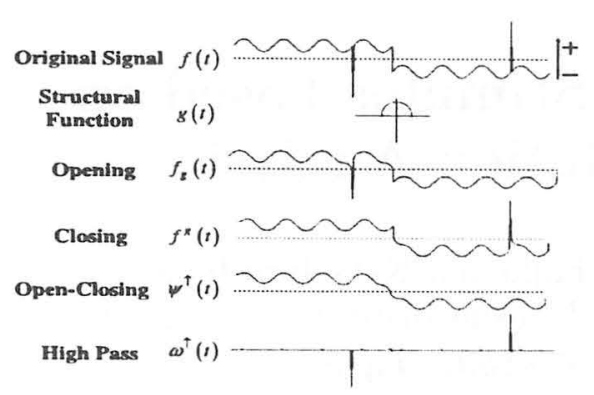

Fig. 1: Property of morphological filter

In other words, opening (resp. closing) smooth $f(t)$ from the positive (resp. negative) direction by $g(t)$. Furthermore, the open-closing filter consisting of successive applications of the opening followed by the closing provides an effect of lowpass filter. Thus, we can also construct a highpass filter by taking the difference between the original signal and its open-closing.

$$
\begin{aligned}
\text { lowpass : } \psi^{\dagger}(t) & =\left(f_{g}\right)^{g}(t) \\
\text { highpass : } \omega^{\dagger}(t) & =f(t)-\psi^{\dagger}(t)
\end{aligned}
$$

The schematic figures of morphological operations are shown in Fig. 1.

\subsection{Design of Structuring Function}

In processing mathematical morphology, the characteristics of filter are decided by the shape of structuring function. By the convention, the structuring function has finite values only in the processing window and takes $-\infty$ on the outside. In this paper, we use the Haar type and SuperEllipse type structuring functions (Fig. 2). Haar type structuring function is defined with width of window $2 n$ as follows.

$$
g(t)= \begin{cases}0 & (-n \leq t \leq n) \\ -\infty & \text { (otherwise) }\end{cases}
$$

On the other hand, SuperEllipse type structuring function is defined with a variable parameter $k$, width of window $2 n$ and curvature parameter $p$ as follows.

$$
g(t)= \begin{cases}k\left(1-\left|\frac{x}{n}\right|^{p}\right)^{\frac{1}{p}} & (-n \leq t \leq n) \\ -\infty & \text { (otherwise) }\end{cases}
$$

These parameters are optimized to become different during separated signals $x_{L}$ and $x_{H}$ by random search. The condition of difference is evaluated by using two methods as follows.

\section{1) Mutual information method}

The heuristic parameters of structuring function are optimized by using mutual information between separated signals $\mathbf{x}^{\mathbf{j}}=\left\{x_{L}^{j}, x_{H}^{j}\right\}$. The evaluation function is the joint entropy between $x_{L}^{j}$ and $x_{H}^{j}$.

$$
H\left(\mathbf{x}^{j}\right)=\frac{1}{2} \log _{2}\left\{(2 \pi e)^{2}|\operatorname{det}(\Sigma)|\right\}
$$

where, $\Sigma$ is covariance matrix of $\mathrm{x}^{\mathrm{j}}$.

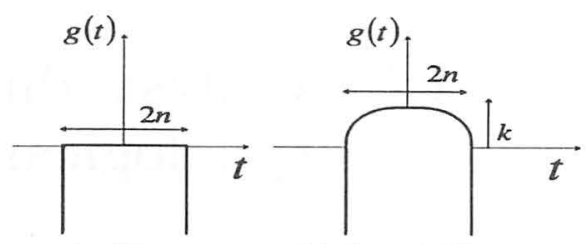

(a) Haar

(b) SuperEllipse

Fig. 2: Model of structuring function

2) Spectrum dividing method

The heuristic parameters are optimized to become separating between signals in frequency domain. In this method, the prior parameter of separation frequency $F_{s p}$, the penalty coefficient $p$ and prior parameters of evaluation interval $F_{\min }$ and $F_{\max }$ need to decide before processing.

$$
\begin{aligned}
C\left(\mathbf{x}^{j}\right) & =\frac{\int_{F_{\min }}^{F_{s p}}\left(\hat{x}_{L}^{j}(f)-p \cdot \hat{x}_{H}^{j}(f)\right) \cdot \hat{x}^{j-1}(f) d f}{F_{s p}-F_{\min }} \\
& +\frac{\int_{F_{s p}}^{F_{\max }}\left(\hat{x}_{H}^{j}(f)-p \cdot \hat{x}_{L}^{j}(f)\right) \cdot \hat{x}^{j-1}(f) d f}{F_{\max }-F_{s p}}
\end{aligned}
$$

where, $x^{j-1}$ is original signal of $\mathbf{x}^{\mathbf{j}}$ and $\hat{\mathbf{x}}^{j}=\left\{\hat{x}_{L}^{j}, \hat{x}_{H}^{j}\right\}$ is Fourier spectrum of $x^{j}$.

\subsection{Multiresolution analysis}

A sequence of successive processes with varying structuring functions constitutes a multiresolution signal analysis. To describe this, let us assume that there exist sets $V_{j}$ at level $j$. In usual wavelet analysis method, for a given input signal $x^{0} \in V_{0}$, we obtain the following recursive analysis scheme:

$$
x^{0} \rightarrow\left\{x_{L}^{1}, x_{H}^{1}\right\} \rightarrow\left\{x_{L L}^{2}, x_{L H}^{2}, x_{H}^{1}\right\} \rightarrow \cdots
$$

This scheme is used for method sequentially extracting from high frequency component. For example, DWT is composed by using details $D(j)$ and smooths $S(j)$ as follows.

$$
x^{0}=\sum_{j=1}^{J} D(j)+S(J)
$$

On the other hand, the full wavelet packet analysis algorithm is used for splitting algorithm.

$$
x^{0} \rightarrow\left\{x_{L}^{1}, x_{H}^{1}\right\} \rightarrow\left\{x_{L L}^{2}, x_{L H}^{2}, x_{H L}^{2}, x_{H H}^{2}\right\} \rightarrow \cdots
$$

In this paper, morphological methods are implemented to latter by using Eqs. (5)(6). A three-level signal analysis scheme is shown in Fig. 3. When non redundant method, the down sampling is processed in each level.

\section{Simulations}

The proposed method was applied to synthesized signal. This signal is composed of sinusoidal wave and white or impulse noise. The sampling frequency is 
$500 \mathrm{~Hz}$. In the morphological multiresolution analysis, the structuring function which decides characteristics of the filter is designed in SuperEllipse and Haar type. Structuring function of SuperEllipse type which composed of three heuristic parameters is designed by using two kinds of optimization method in Eqs. (9)(10) (Fig. 4). Haar type is designed by increasing the width of windows by 2 to the power of levels. The suitable heuristic parameters are obtained by the random search about 1000 times. Especially, spectrum dividing method needs the decide prior parameters such as $F_{\min }, F_{\max }, F_{s p}$ and $p$ in Eq. (10). On the other hand, in comparison with wavelet analysis, we employ Daubechies 6 as the mother.

One result of mutual information method is shown in Fig. 5. This signal is composed of two kinds of sinusoidal waves ( $5 \mathrm{~Hz}$ and $20 \mathrm{~Hz}$ ) and white noise. In this case, the SNR is 7.98 and each component is separated each other. On the other hand, separation of spectrum dividing method is more robust to white noise than $\mathrm{mu}$ tual information method has (Table 1). This analysis result is presented in Fig. 6 (SNR: -1.57). In case of Haar type structuring function, analysis of five levels is necessary to separate all components in this situation (Fig. 7). The component of $20 \mathrm{~Hz}$ was rarely extracted when signal is synthesized with much white noise (SNR is -1.57$)$. In contrast, wavelet analysis is able to extract

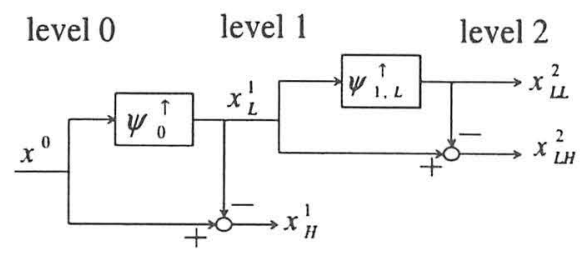

(a) Usual wavelet analysis

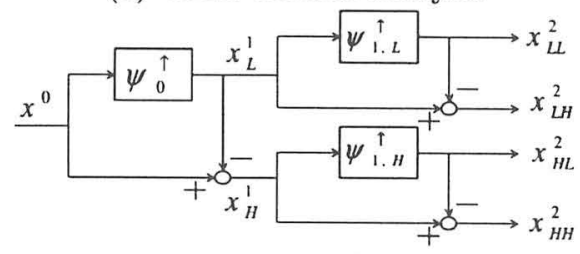

(b) Full wavelet analysis

Fig. 3: Signal analysis scheme

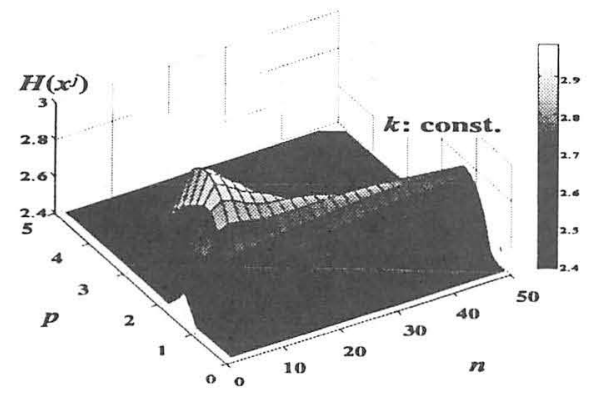

Fig. 4: Criterion Map

The value of mutual information is changed according to the parameter of structuring function. The parameters that compose maximum value are chosen by random search.

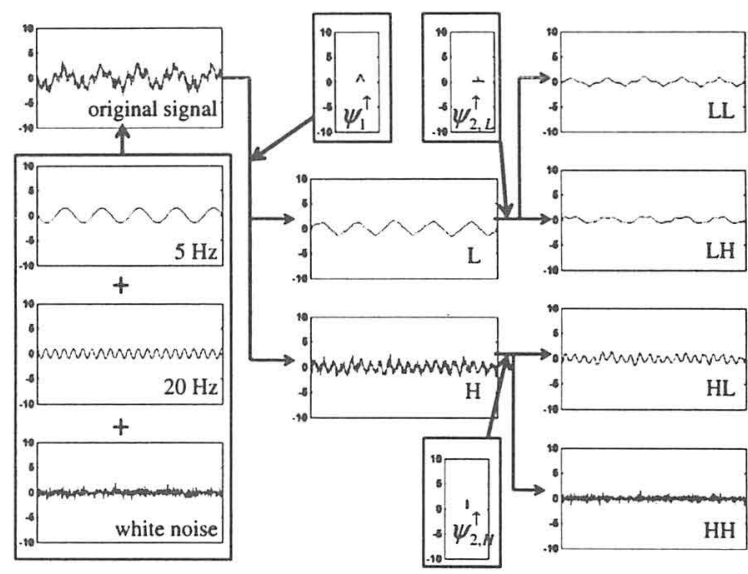

Fig. 5: Analysis of less-noisy synthesized sinusoidal wave

(method: Mutual Info., SNR: 7.98, repeat count: 10k)
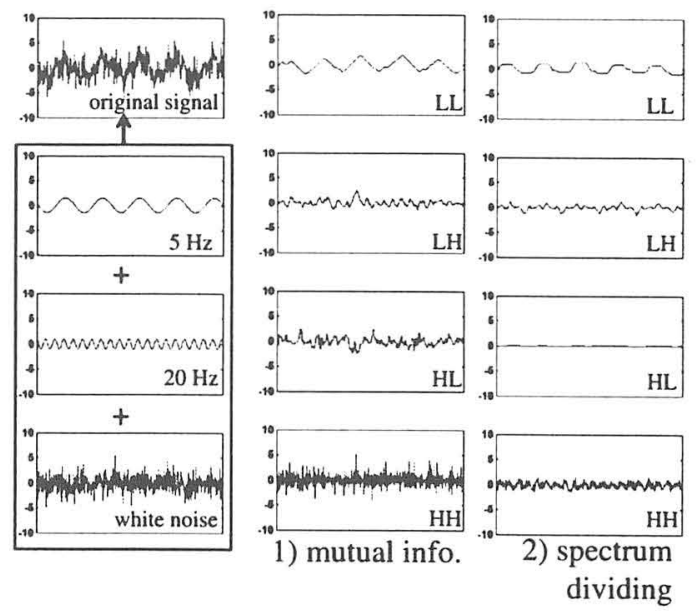

Fig. 6: Analysis of noisy synthesized sinusoidal wave (SNR: $-1.57, \mathrm{SR}: 500 \mathrm{~Hz}$, repeat count: $1000, p=0.2$, $F_{\min }=2 \mathrm{~Hz}, F_{\max }=30 \mathrm{~Hz}, F_{s p}=23,17,30 \mathrm{~Hz}$ )

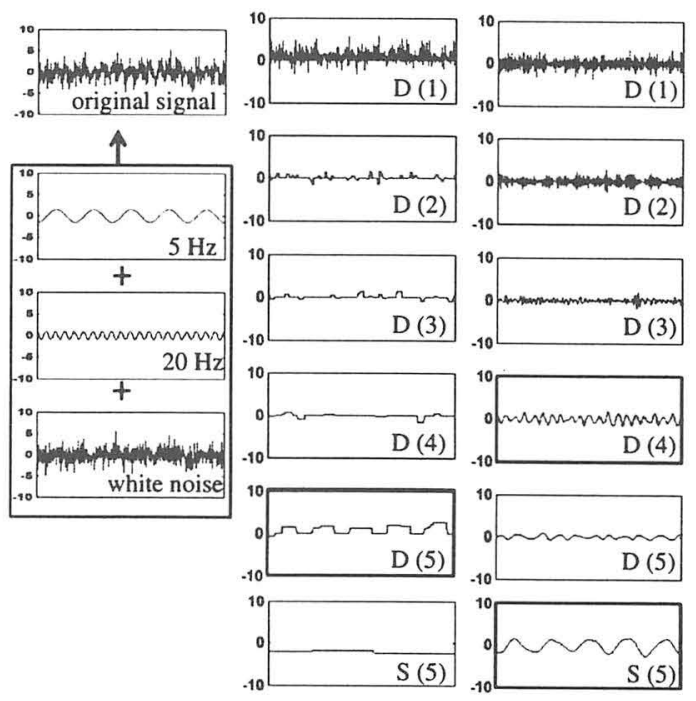

Fig. 7: Comparison during wavelet and morphology (Left: morphology (Haar), Right: Wavelet (Daub. 6)) 
all components effectively. The coefficient of correlation between input signal and analysed signal is higher than the case of morphological method (wavelet (Daubechies 6): 0.87, morphology (Haar): 0.44). When the impulse noise is mixed with sinusoidal wave $(17 \mathrm{~Hz})$, morphological method has provided components effectively (Fig. 8). The impulse component was extracted in a few bands and the distortion is not caused form impulse response (Table 2).

These results suggest that, in a certain field, the morphological method with the optimized structural function is more suitable to extract features from signal. Especially, the mutual information method is useful when components are not understood beforehand because the prior parameters are not necessary (Table 3 ).

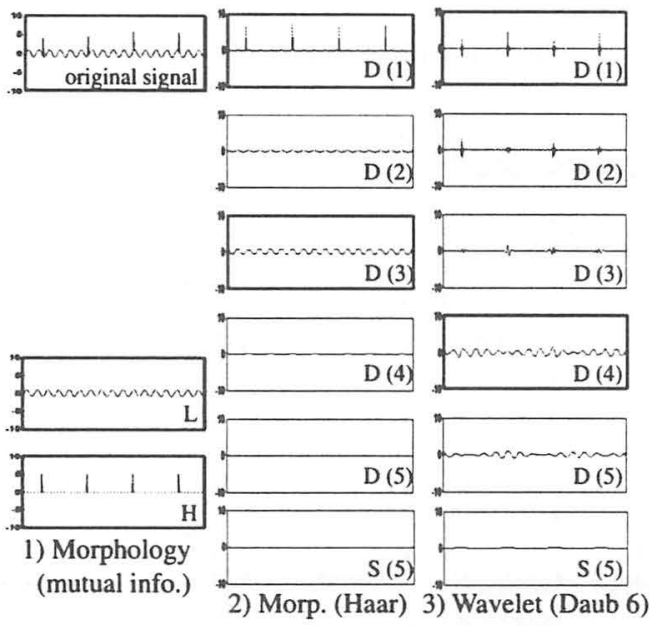

Fig. 8: Multiresolution analysis to impulse signal 1) Morphology (mutual info.): Pulse and sinusoidal components are divided at one level clearly. 2) Morphology (Haar): Though pulse component is extracted in $\mathrm{D}(1)$, sinusoidal component appear in $\mathrm{D}(2)$ and $\mathrm{D}(3)$. 3) Wavelet (Daub 6): Component of impulse response appear in $\mathrm{D}(2)$ and $\mathrm{D}(3)$, and shape of wave is strained.

Table 1: Coefficient of correlation compared with original signals (white noise)

\begin{tabular}{ccccc}
\hline & & & & SNR: 7.98 \\
\hline & M-MI & M-SD & M-HA & W-D6 \\
\hline $5 \mathrm{~Hz}$ & 0.94 & 0.91 & 0.89 & 0.98 \\
$20 \mathrm{~Hz}$ & 0.91 & 0.85 & 0.67 & 0.95 \\
white noise & 0.88 & 0.82 & 0.76 & 0.73 \\
\hline & & & & SNR: -1.57 \\
\hline & M-MI & M-SD & M-HA & W-D6 \\
\hline $5 \mathrm{~Hz}$ & 0.91 & 0.88 & 0.80 & 0.95 \\
$20 \mathrm{~Hz}$ & 0.57 & 0.42 & 0.44 & 0.87 \\
white noise & 0.85 & 0.90 & 0.86 & 0.73 \\
\hline
\end{tabular}

M-MI: Morphology (Mutual Information of SuperEllipse)

M-SD: Morphology (Spectrum Dividing of SuperEllipse)

M-HA: Morphology (Haar type)

W-D6: Wavelet (Daubechies 6)
Table 2: Coefficient of correlation and MSE compared with original signals (impulse noise)

\begin{tabular}{cccc}
\hline & & & SNR: 4.01 \\
\hline & & & $\rho$ \\
\hline & M-MI & M-HA & W-D6 \\
\hline $17 \mathrm{~Hz}$ & 0.99998 & 0.98219 & 0.80526 \\
impulse noise & 0.99997 & 0.99704 & 0.70995 \\
\hline \hline \multicolumn{5}{c}{} & & & MSE \\
\hline $17 \mathrm{~Hz}$ & 0.00002 & 0.08839 & 0.17855 \\
impulse noise & 0.00002 & 0.00233 & 0.19600 \\
\hline
\end{tabular}

M-MI: Morphology (Mutual Information of SuperEllipse)

M-HA: Morphology (Haar type)

W-D6: Wavelet (Daubechies 6)

( $\rho$ : coefficient of correlation, MSE: mean square error)

Table 3: Specification of the methods

\begin{tabular}{ccc}
\hline & white noise & impulse noise \\
\hline Morp. (Mutual) & $\triangle$ & $\bigcirc$ \\
Morp. (Spectrum) & $\triangle *$ & $\bigcirc$ \\
Morp. (Haar) & $\times$ & $\bigcirc$ \\
Wavelet (Daub 6) & $\bigcirc$ & $\times$ \\
\hline (*: Need to tune of the prior parameters)
\end{tabular}

\section{Application for BCI System}

\subsection{Experimental system}

Three subjects $(\mathrm{X}, \mathrm{Y}, \mathrm{Z})$ participated in this study for three days. EEG signal is measured from occipital area $(\mathrm{Oz}-\mathrm{A} 2)$ with $512 \mathrm{~Hz}$ sampling frequency. Each trial consist of two kinds of visual stimulus pattern (Pattern $\mathrm{A}, \mathrm{B}$ ) that is presented either one during $1000 \mathrm{~ms}$ (Fig. 9). During the experiment, the subject fixated a computer monitor $100 \mathrm{~cm}$ in front of him and gazed to visual stimulation. This experiment was examined about 800 trials in each day.

\subsection{Pattern recognition methods}

Fig. 10 shows averaged signals during stimulus and non-stimulus. The two kinds of difference between stimulus and non-stimulus are confirmed at $75 \mathrm{msec}$ and $100 \mathrm{msec}$ (these are called P75 and N100). It is known that P75 takes positive potential and N100 takes negative potential. The decomposition results of single trial EEG is depicted in Fig. 11. In case of pattern A, a negative peak concerned with 5 th stimulus appeared at $100 \mathrm{~ms}$ delay of level LH. Similarly, the negative peak concerned with 4th stimulus appeared in case of pattern B. Distribution of the peak latency and this amplitude while one experiment is shown in Fig 12. It is confirmed that most of these parameters relates to event. We assume this amount of potential follows in each section normal distribution. The decision rule of pattern $\mathrm{A}$ or 


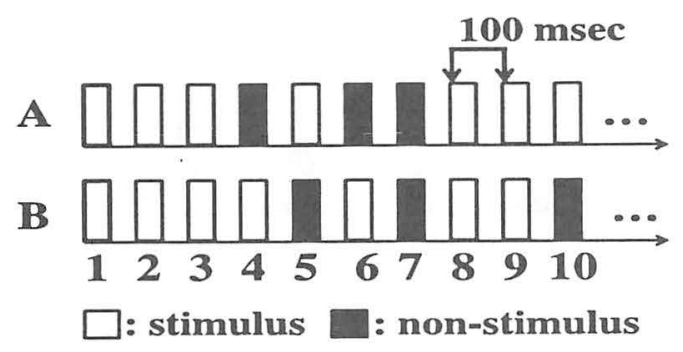

Fig. 9: Timing chart

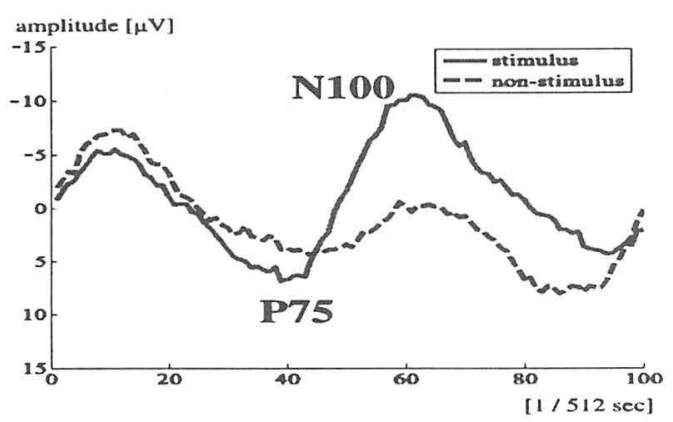

Fig. 10: Averaged signal
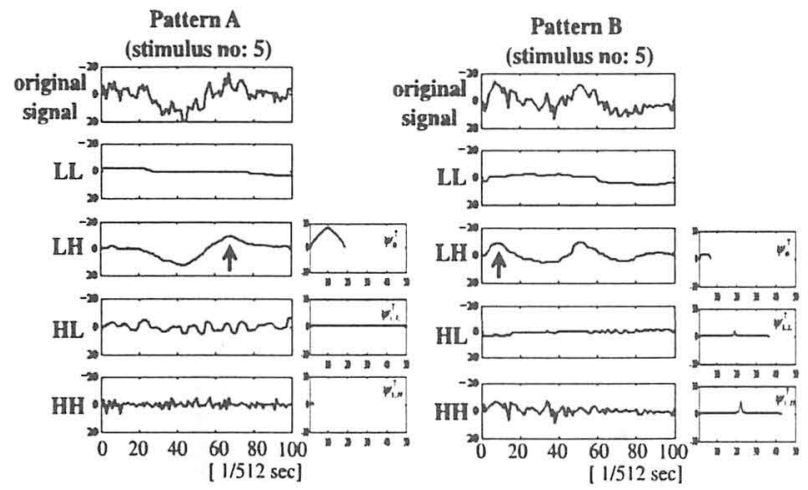

Fig. 11: Decomposition of single trial EEG

EEG signals are decomposed to two levels by using three kinds of structuring function. Depending on stimulus pattern, N100 component are extracted at the time of corresponding latency.

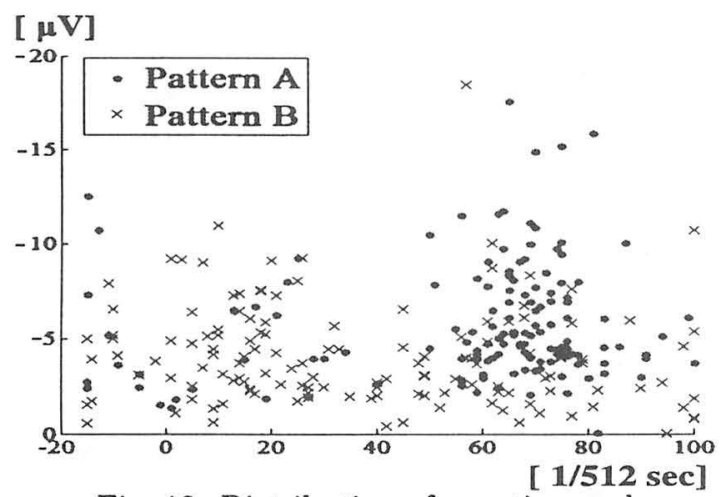

Fig. 12: Distribution of negative peak

(Level: LH, stimulus no: 5 , subject: $\mathrm{X}$, session no: 1 ) Latency and amplitude of negative peak which confirmed in Fig. 11 are distributed like this figure. The processing period of Eq. (12) ( $d$ and $w$ ) are decided according to this tendency.
$\mathrm{B}$ is composed as follows,

$$
\begin{aligned}
k^{*} & =\arg \max _{k} \operatorname{Pr}\left(\omega_{k} \mid \mathbf{s}\right) \\
& =\arg \min _{k}\left\{\frac{1}{2} \ln \left|\Sigma_{k}\right|\right. \\
& \left.+\frac{1}{2}\left(\mathbf{s}-\mathbf{m}_{k}\right)^{T} \Sigma_{k}^{-1}\left(\mathbf{s}-\mathbf{m}_{k}\right)-\operatorname{Pr}\left(\omega_{k}\right)\right\} \\
& \left(\begin{array}{c}
s_{1}=\sum_{t=d-w}^{d+w}\{y(t+500)+y(t+1000)\}, \\
s_{2}=\sum_{t=d-w}^{d+w}\{y(t+400)+y(t+600)\}
\end{array}\right)
\end{aligned}
$$

where, $y$ is output of morphological filter and $\mathbf{m}_{k}$ is mean and $\Sigma_{k}$ is variance of $\mathbf{s}$. Also, $d$ is latency of P75 and $\mathrm{N} 100$ and $w$ is processing window.

\section{Results}

EEG signals are separated by using mutual information method. A single trial EEG signal are decomposed at two levels by morphological multiresolution analysis. At the time just after presentation of light, the peak is observed at a specific level, it is P75 and N100. The criterion $\mathbf{s}$ which is used at Eq. 12 is distributed such as Fig. 13. It seems to be able to distinguish the gazed pattern. These features are evaluated by cross validation method (ratio of training data:test data $=4: 1$ ). The parameter $w$ is selected to 5 and parameters of structuring function are optimized by 1000 times random search.

Figs. 15, 16 show pattern recognition results of analyzed signals by morphological method and wavelet in P75, N100 and both. The morphological method could decompose without lack of information too much. Especially, the recognition accuracy using P75 feature was improved. On the other hand, wavelet method divides effective information for recognition. Therefore, it is thought that the morphological method is more useful to extract principal component of VEP. The optimized structuring functions in this case are depicted in Fig. 14. In case of subject $\mathrm{X}$, the structuring function is composed to sharp shape and high accuracy is obtained at level LH, HL and HH. Contrary, the high accuracy observed to level LL, LH and HL when rounded shape is estimated (subjects $\mathrm{Y}$ and $\mathrm{Z}$ ). This phenomenon suggest that the optimized structuring functions take characteristics of signal structure. These characteristics are useful to specify features from signal.

\section{Conclusion}

In this paper, feature extraction method based on morphological multi-resolution analysis was introduced to extract features concerned with visual evoked potential from EEG signals. The pattern which subject pays attention can be distinguished by using the EEG data about one sec. The morphological method is superior to 
wavelet method in feature extraction from visual evoked potential in the meaning of identifying the characteristic band. It seems that the essential features are able to be identified by decomposing signal without lack of information for the pattern recognition. Furthermore, the estimated structuring function has the possibility of containing characteristics of original signal. An efficient P300 typewriter system will be able to be achieved by advancing this research further.

\section{References}

[1] C. Guger, S. Daban, E. Sellers, C. Holzner, G. Krausz, R. Carabalona, F. Gramatica, and G. Edlinger. " How many people are able to control a P300-based brain-computer interface (BCI)?". Neuroscience Letters, 462:94-98, 2009.

[2] H. Kobatake. "Morphology". CORONA PUBLISHING CO., LTD., 1996.

[3] S. G. Mallat. "Zero-crossings of a wavelet transform". IEEE Transaction on Information Theory, 37-4:1019-1033, 1991.

[4] G. Pfurtscheller, C. Brunner, A. Schlögl, and F. L. da Silva. "Mu rhythm (de)synchronization and EEG single-trial classification of different motor imagery tasks". NeuroImage, 31-1:153-159, 2006.

[5] J. J. Vidal. "Toward direct brain computer communication". Annual Rev. Biophysics Bio-engineering, pages 157-180, 1973.

[6] J. Wolpaw and D. McFarland. "Multichannel EEG based brain computer communications". Electroencephalography and clinical Neuro-phyiology, 90:444449, 1997.

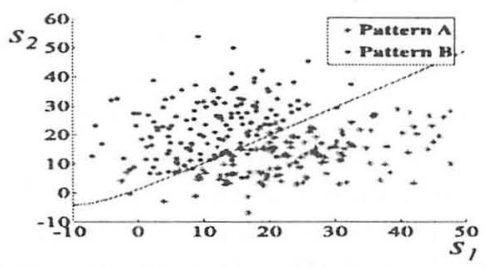

Fig. 13: Distribution of criterion

(Subj.: B, Session no: 2, channel: Oz, feature: N100 )

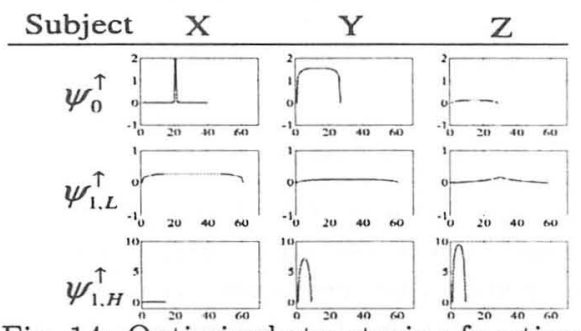

Fig. 14: Optimized structuring functions (Session no: 2, channel: $\mathrm{Oz}$ )

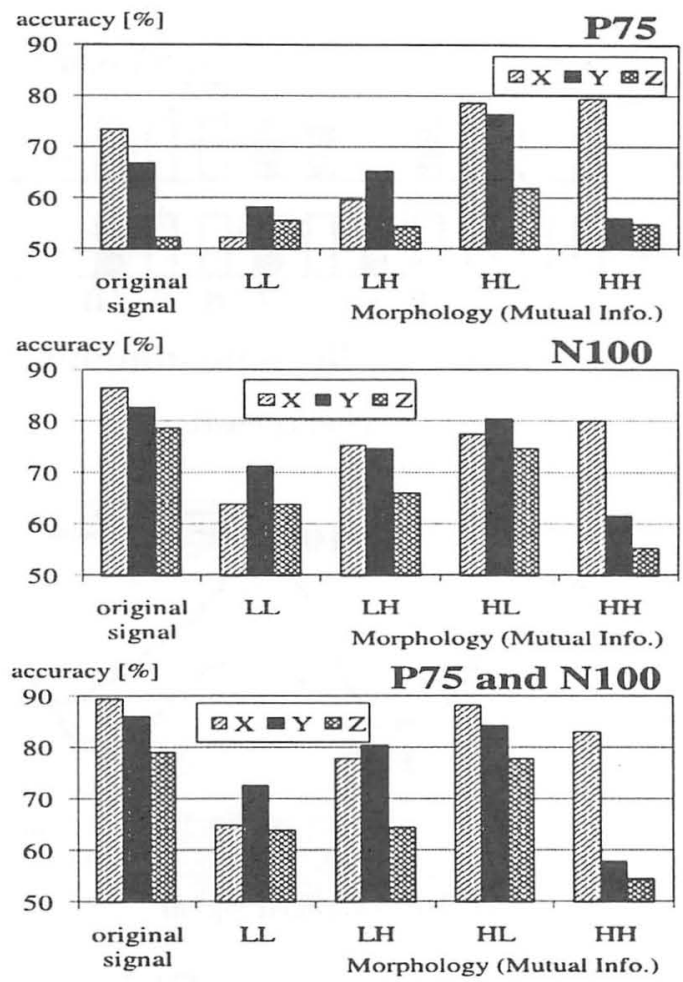

Fig. 15: Pattern recognition results by using morphological MRA

(Session no: 2, channel: $\mathrm{Oz}$ )
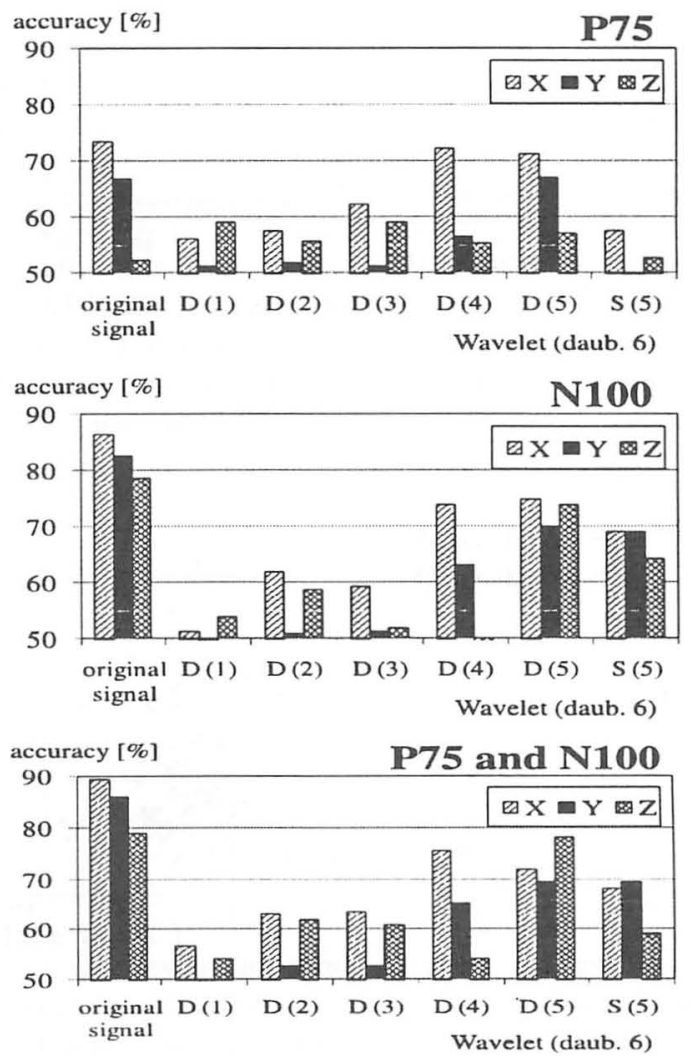

Fig. 16: Pattern recognition results by using wavelet (Session no: 2, channel: $\mathrm{Oz}$ ) 